\title{
Dynamic PMSM Motor Model with Turn Short Fault for Wind Power Generation
}

\author{
Bon-Gwan $\mathrm{Gu}^{1}$, Jun-Hyuk Choi ${ }^{1}$, and In-Soung Jung ${ }^{1}$ \\ ${ }^{1}$ Korea Electronics Technology Institute \\ 203-101 Bucheon-TP B/D 192, \\ Yakdae-dong, Wonmi-gu,Bucheon-si, Gyeonggi-do (South Korea) \\ Phone number: +82)-32-621-2864, e-mail: mrgu@ postech.ac.kr
}

\begin{abstract}
A PMSM (Permanent Magnet Synchronous Motor) stator inter turn short fault generates the back emf and impedance unbalance element. Those unbalance element cause the torque ripple and the escalating partial heat defect. This paper proposes a dynamic motor model under the internal turn short fault. The proposed model is derived in the positive and negative sequence synchronous reference frame to be easily utilized for the fault detection rule. To validate the proposed scheme, the finite elements method (FEM) simulation with electric circuit is conducted on the PMSM.
\end{abstract}

\section{Key words}

PMSM (Permanent magnet synchronous motor), turn short fault, fault model.

\section{Introduction}

The reliability is one of the important issue in the wind power generation system since it affects directly the idle time cost. Hence, every wind power system part has to be trouble free and fault-detectable. Hence, the PMSM, which has been widely used for the generator of the wind power generation system, also need to be reliable and easily fault detectable.

One common fault of the PMSM is a winding turn short fault caused by the coil insulation breakdown. Since the coil insulation material is under the high voltage and temperature stress, it degrades gradually and reaches to the inter turn short fault. When the inter turn short happens, the shorted turns composes an extra circuit loop which is coupled with others motor winding flux and the rotor magnet flux. The couple flux induces the high fault current in the turn fault winding. The high fault current generates ohmic losses and heats up the shorted winding. The heat is conducted to the near winding and weakens the insulation material. Hence, the inter turn fault easily expands to the near winding.

When the inter turn short occurs, the phase impedance and the back emf are reduced in the turn short phase winding. Hence, the impedance and back emf of the three phase winding are not balanced. As a result, the negative sequence terms are generated in the motor current or the

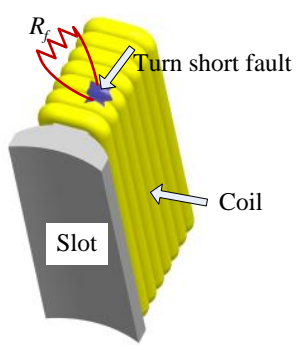

(a)

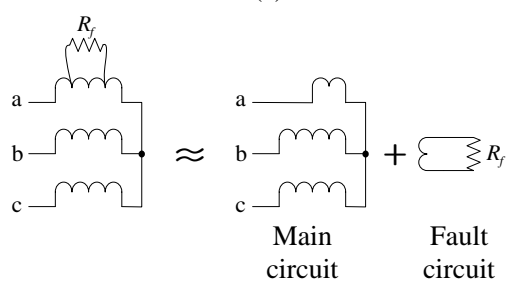

(b)

Fig. 1.Motor coil with turn short fault, (a) motor slots and turn short fault, (b) equivalent circuit model and simplified two circuit model

voltage [1]-[4]. Note further that the normal fieldoriented control yields torque ripple at the presence of negative sequence terms. In the previous works, the inter turn fault were developed mostly in the abc-frame [2]-[8]. They are complex since they include the negative and the positive sequences. One previous work has shown in the d-q reference frame [1]. The dynamic model is simple and easy to implement in the filed-oriented control scheme. But, it has focused on the healthy winding excluding the faulted inter turn loop.

In this paper, dynamic d-q model of the PMSM under the inter turn short fault is derived in the positive and the negative sequence rotating frame. With the model, the fault detection scheme can be easily developed.

\section{Dynamic PMSM Model under Turn Short Fault}

Fig. 1 (a) shows the winding configuration of the motor under the internal turn short. $R_{f}$ denotes a contact resistance of the turns short fault. The turn short fault winding makes another fault circuit loop which is 

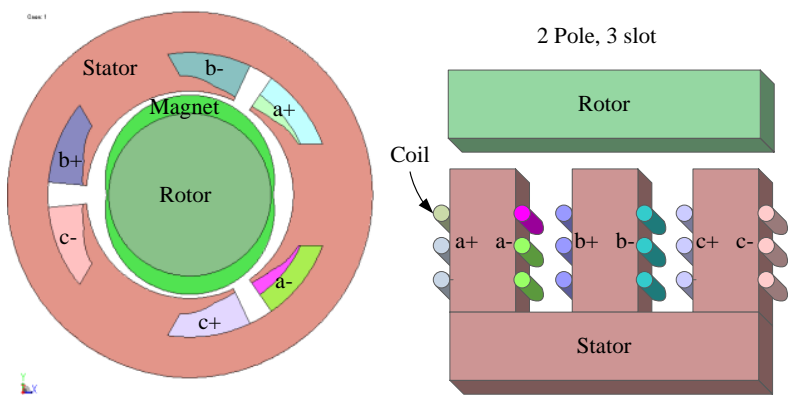

(a)

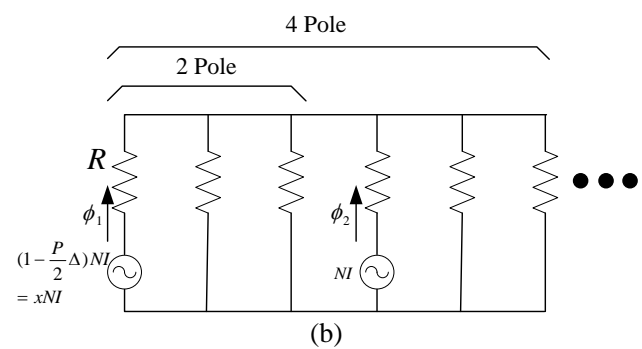

Fig. 2. (a) 2poles-3slots PMSM motor cross-sectional figure and simplified model for inductance calculation, (b) equivalent magnetic circuit for inductance calculation.

composed of the contact resistance, fault winding, and back emf. The fault circuit loop is connected to the main circuit. The overall circuit analysis is very complicated. Hence, we divide the PMSM motor model into a main circuit and a fault circuit. The main circuit and the fault circuit can be modeled as shown in Fig. 1 (b). Since they are not electrically connected to each other, the whole analysis is much simpler. But, they are coupled magnetically, the cross coupling effects should be considered in the analysis.

\section{A. Main circuit model}

The turn number of main circuit a-phase is reduced by the fault. Hence, the motor model changes depending on the faulted turn number. To establish the main circuit model, the equivalent magnetic circuit is used. Fig. 2 (a) shows a cross-sectional figure and a simplified model of 2pole PMSM with the concentric winding. In the left figure, the a-phase winding is divided into two parts, i.e., healthy winding and fault winding. Fig. 2 (b) shows the magnetic circuit for inductance calculation of $2,4, \ldots$ pole PMSM, where $\phi_{1,2, . . \mathrm{P} / 2}, N, P$, and $R$ denote the magnetic flux of the slots, the turn number of one slot, the pole number, and the magnetic reluctance, respectively. $x$ and $\Delta$ denote the rate of the healthy turn rate in one slot and the rate of the fault turn of one phase. $x$ and $\Delta$ satisfy

$$
\Delta=N_{\text {short }} / P N=(1-x) /(P / 2),
$$

where $N_{\text {short }}$ denotes the fault turn number. By the magnetic circuit of Fig. 2(b), the flux density at the faulted phase is given by,

$$
\begin{gathered}
\phi_{1}=\frac{x N I\left(\frac{3}{2} P-1\right)-N I\left(\frac{P}{2}-1\right)}{R\left(\frac{3}{2} P-1\right)+R}, \\
\phi_{2,3, . . P / 2}=\frac{N I\left(\frac{3}{2} P-1\right)-N I\left(\frac{P}{2}-2\right)-x N I}{R\left(\frac{3}{2} P-1\right)+R} .
\end{gathered}
$$

By eq. (2) and (3), the self magnetizing inductance $L_{m}$ can be obtained such that

$$
\begin{aligned}
L_{m} & =\frac{x N \phi_{1}}{I}+\frac{N \phi_{2}}{I}+\ldots \frac{N \phi_{P / 2}}{I}=\frac{x N \phi_{1}+N \phi_{2}(P / 2-1)}{I} \\
& =\frac{N^{2}}{R} \frac{2}{3 P}\left(\left(\frac{3}{2} P-1\right) x^{2}+(2-P) x+\frac{P^{2}}{2}-\frac{P}{2}-1\right) \\
& =\frac{P N^{2}}{3 R}\left(\left(\frac{3 P}{4}-\frac{1}{2}\right) \Delta^{2}-2 \Delta+1\right)
\end{aligned}
$$

If the PMSM has no fault $(\Delta=0)$, the self magnetizing inductance is $L_{m}=P N^{2} / 3 R$. But, if the PMSM has an internal turn short $(1 \geq \Delta>0)$, the decrease of the self magnetizing inductance has a quadratic function.

With eq. (4), the flux linkage of the PMSM under the a-phase winding inter turn fault is given by

$$
\lambda_{a b c}=\mathbf{L}_{a b c} \mathbf{i}_{a b c}+\boldsymbol{\psi}_{a b c}=\left(\mathbf{L}_{s y m}+\mathbf{L}_{n s y m}\right) \mathbf{i}_{a b c}+\boldsymbol{\psi}_{a b c},
$$

where $\lambda_{a b c}=\left[\begin{array}{lll}\lambda_{a} & \lambda_{b} & \lambda_{c}\end{array}\right]^{T}, \mathbf{i}_{a b c}=\left[\begin{array}{lll}i_{a} & i_{b} & i_{c}\end{array}\right]^{T}$,

$$
\boldsymbol{\psi}_{a b c}=\psi_{m}\left[\begin{array}{ll}
(1-\Delta) \cos \theta & \cos \left(\theta-\frac{2 \pi}{3}\right) \\
\cos \left(\theta+\frac{2 \pi}{3}\right)
\end{array}\right]^{T},
$$

$$
\begin{gathered}
\mathbf{L}_{s y m}=\left[\begin{array}{ccc}
L_{m}+L_{l} & -\frac{1}{2} L_{m} & -\frac{1}{2} L_{m} \\
-\frac{1}{2} L_{m} & L_{m}+L_{l} & -\frac{1}{2} L_{m} \\
-\frac{1}{2} L & -\frac{1}{2} L_{m} & L_{m}+L_{l}
\end{array}\right] \\
\mathbf{L}_{n s y m}=\left[\begin{array}{ccc}
-L_{f} & \frac{\Delta}{2} L_{m} & \frac{\Delta}{2} L_{m} \\
\frac{\Delta}{2} L_{m} & 0 & 0
\end{array}\right] \\
L_{f}=-\left(\Delta^{2}\left(\frac{3 P}{2}-\frac{1}{2}\right)-2 \Delta\right)\left(L_{m}+L_{l}\right) .
\end{gathered}
$$

$\psi_{m}$ denotes the permanent magnet flux linkage. Since $\mathbf{L}_{n s y m}$ and $\boldsymbol{\psi}_{a b c}$ have the unbalanced coupling terms, the flux linkage, the phase current, and the phase voltage have the negative sequence elements. By the stationary dq reference frame transformation of (5), the positive and the negative sequence flux linkage $\lambda_{d q s}^{+}, \lambda_{d q s}^{-}$are derived as 


$$
\begin{aligned}
\lambda_{d q s}^{+} & =L_{s} \mathbf{I}_{d q s}^{+}+\psi_{m}\left(1-\frac{\Delta}{3}\right) e^{\mathrm{J} \theta}+\frac{2}{3} \Delta_{m} I_{d s}^{+}, \\
\lambda_{d q s}^{-} & =L_{s} \mathbf{I}_{d q s}^{-}-\psi_{m} \frac{\Delta}{3} e^{-\mathbf{J} \theta}+\frac{2}{3} \Delta_{m} I_{d s}^{-},
\end{aligned}
$$

where $\lambda_{d q s}^{+}=\left[\begin{array}{ll}\lambda_{d s}^{+} & \lambda_{q s}^{+}\end{array}\right]^{T}, \mathbf{I}_{d q s}^{+}=\left[\begin{array}{ll}I_{d s}^{+} & I_{d s}^{+}\end{array}\right]^{T}$,

$$
\begin{aligned}
\lambda_{d q s}^{-} & =\left[\begin{array}{ll}
\lambda_{d s}^{-} & \lambda_{q s}^{-}
\end{array}\right]^{T}, \mathbf{I}_{d q s}^{+}=\left[\begin{array}{ll}
I_{d s}^{-} & I_{d s}^{-}
\end{array}\right]^{T}, \\
L_{s} & =1.5 L_{m}+L_{l}, \\
\Delta_{m} & =\left(\Delta^{2}\left(\frac{3 P}{4}-\frac{1}{2}\right)-3 \Delta\right) L_{m}+\left(\Delta^{2}\left(\frac{3 P}{4}-\frac{1}{2}\right)-2 \Delta\right) L_{l} \\
e^{\mathbf{J} \theta} & =\left[\begin{array}{cc}
\cos \theta & -\sin \theta \\
\sin \theta & \cos \theta
\end{array}\right] .
\end{aligned}
$$

$\mathbf{I}_{d q s}^{+}, \mathbf{I}_{d q s}^{-}$, and $\theta$ denote the positive and the negative sequence current vector in the stationary $d-q$ reference frame, and rotor position, respectively. In a Synchronous Reference Frame (SRF), the derivatives of the positive and the negative sequence flux linkage $\lambda_{d q e}^{+}, \lambda_{d q e}^{-}$are derived as

$$
\begin{aligned}
& \frac{d \lambda_{d q e}^{+}}{d t}=\omega \mathbf{L}_{s} \mathbf{J I}_{d q e}^{+}+L_{s} \frac{d \mathbf{I}_{d q e}^{+}}{d t}+\frac{d \mathbf{L}_{\alpha} \mathbf{I}_{d q e}^{+}}{d t}+\omega \psi_{m}\left(1-\frac{\Delta}{3}\right)\left[\begin{array}{l}
0 \\
1
\end{array}\right] \\
& \frac{d \lambda_{d q e}^{-}}{d t}=-\omega \mathbf{L}_{s} \mathbf{J I}_{d q e}^{-}+L_{s} \frac{d \mathbf{I}_{d q e}^{-}}{d t}+\frac{d \mathbf{L}_{\beta} \mathbf{I}_{d q e}^{-}}{d t}+\omega \psi_{m}\left(\frac{\Delta}{3}\right)\left[\begin{array}{l}
0 \\
1
\end{array}\right]
\end{aligned}
$$

where $\mathbf{I}_{d q e}^{+}=e^{-\mathbf{J} \theta} \mathbf{I}_{d q s}^{+}, \mathbf{I}_{d q e}^{-}=e^{\mathbf{J} \theta} \mathbf{I}_{d q s}^{-}$,

$$
B=\frac{2 \Delta_{m}}{3}\left[\begin{array}{ll}
1 & 0 \\
0 & 0
\end{array}\right], \quad \mathbf{L}_{\alpha}=e^{-\mathbf{J} \theta} \mathbf{B} e^{\mathbf{J} \theta}, \mathbf{L}_{\beta}=e^{\mathbf{J} \theta} \mathbf{B} e^{-\mathbf{J} \theta} .
$$

$\omega$ denotes the rotor speed. The third term on the right hand side of (8) and (9) can be calculated as follows

$$
\begin{aligned}
& \frac{d \mathbf{L}_{\alpha} \mathbf{I}_{d q e}^{+}}{d t}=\frac{d \mathbf{L}_{0 \alpha}}{d t} \mathbf{I}_{d q e}^{+}+\omega \mathbf{L}_{\alpha} \mathbf{J I}_{d q e}^{+}+\mathbf{L}_{\alpha} \frac{d \mathbf{I}_{d q e}^{+}}{d t} \\
= & \frac{\Delta_{m} \omega}{3}\left[\begin{array}{cc}
-\sin 2 \omega t & -\cos 2 \omega t \\
-\cos 2 \omega t & \sin 2 \omega t
\end{array}\right] \mathbf{I}_{d q e}^{+}+\frac{\Delta_{m} \omega}{3} \mathbf{J I}_{d q e}^{+}+\mathbf{L}_{\alpha} \frac{d \mathbf{I}_{d q e}^{+}}{d t} \\
& \frac{d \mathbf{L}_{\beta} \mathbf{I}_{d q e}^{-}}{d t}=\frac{d \mathbf{L}_{\beta}}{d t} \mathbf{I}_{d q e}^{-}-\omega \mathbf{L}_{\beta} \mathbf{J I}_{d q e}^{-}+\mathbf{L}_{\beta} \frac{d \mathbf{I}_{d q e}^{-}}{d t} \\
= & \frac{\Delta_{m} \omega}{3}\left[\begin{array}{cc}
-\sin 2 \omega t & \cos 2 \omega t \\
\cos 2 \omega t & \sin 2 \omega t
\end{array}\right] \mathbf{I}_{d q e}^{-}-\frac{\Delta_{m} \omega}{3} \mathbf{J I}_{d q e}^{-}+\mathbf{L}_{\beta} \frac{d \mathbf{I}_{d q e}^{-}}{d t}
\end{aligned}
$$

The first terms on the right hand side of (10) and (11) have the second order sinusoidal coefficients in the positive and the negative SRF. These two terms are the negative and the positive sequence terms in the positive and the negative SRF. They should be transformed to each other SRF. With two terms transformations and (8)-(11), the dynamic equations of the main circuit are obtained such that

$$
\begin{aligned}
\mathbf{V}_{d q e}^{+} & =r \mathbf{I}_{d q e}^{+}+\frac{d}{d t} \lambda_{d q e}^{+} \\
= & r \mathbf{I}_{d q e}^{+}+\omega L_{S} \mathbf{J I}_{d q e}^{+}+L_{S} \frac{d}{d t} \mathbf{I}_{d q e}^{+}+\omega\left(1-\frac{\Delta}{3}\right) \psi_{m}\left[\begin{array}{l}
0 \\
1
\end{array}\right] \\
& +\frac{\Delta_{m} \omega}{3} \mathbf{J I}_{d q e}^{+}+\frac{\Delta_{m} \omega}{3}\left[\begin{array}{ll}
0 & 1 \\
1 & 0
\end{array}\right] \mathbf{I}_{d q s}^{-}+\mathbf{L}_{\alpha} \frac{d \mathbf{I}_{d q e}^{+}}{d t} \\
\mathbf{V}_{d q e}^{-} & =r \mathbf{I}_{d q e}^{-}+\frac{d}{d t} \lambda_{d q e}^{-} \\
= & r \mathbf{I}_{d q e}^{-}-\omega L_{S} \mathbf{J I}_{d q e}^{-}+L_{S} \frac{d}{d t} \mathbf{I}_{d q e}^{-}+\omega \frac{\Delta}{3} \psi_{m}\left[\begin{array}{l}
0 \\
1
\end{array}\right] \\
& -\frac{\Delta_{m} \omega}{3} \mathbf{J I}_{d q e}^{-}+\frac{\Delta_{m} \omega}{3}\left[\begin{array}{cc}
0 & -1 \\
-1 & 0
\end{array}\right] \mathbf{I}_{d q e}^{+}+\mathbf{L}_{\beta} \frac{d}{d t} \mathbf{I}_{d q e}^{-} .
\end{aligned}
$$

(12) and (13) are the dynamic equation of the main circuit in the positive sequence and the negative sequence SRF.

\section{B. Fault circuit model}

To analyze the fault circuit, the overall flux linkage equation including the fault winding is given by

$$
\boldsymbol{\lambda}_{a b c f}=\mathbf{L}_{a b c f} \mathbf{i}_{a b c f}+\boldsymbol{\psi}_{a b c f},
$$

where $\lambda_{a b c f}=\left[\begin{array}{llll}\lambda_{a} & \lambda_{b} & \lambda_{c} & \lambda_{f}\end{array}\right]^{T}, \mathbf{i}_{a b c f}=\left[\begin{array}{llll}i_{a} & i_{b} & i_{c} & i_{f}\end{array}\right]^{T}$,

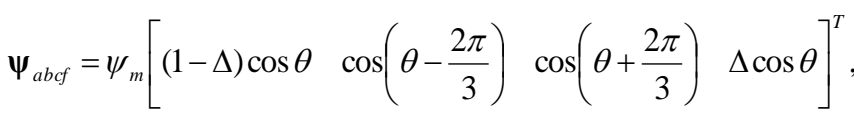

$\mathbf{L}_{a b c f}=\left[\begin{array}{cccc}L_{m}+L_{l}-L_{f} & -\frac{1-\Delta}{2} L_{m} & -\frac{1-\Delta}{2} L_{m} & L_{f}-\Delta\left(L_{m}+L_{l}\right) \\ -\frac{1-\Delta}{2} L_{m} & L_{m}+L_{l} & -\frac{1}{2} L_{m} & -\frac{\Delta}{2} L_{m} \\ -\frac{1-\Delta}{2} L_{m} & -\frac{1}{2} L_{m} & L_{m}+L_{l} & -\frac{\Delta}{2} L_{m} \\ L_{f}-\Delta\left(L_{m}+L_{l}\right) & -\frac{\Delta}{2} L_{m} & -\frac{\Delta}{2} L_{m} & -L_{f}+2 \Delta\left(L_{m}+L_{l}\right)\end{array}\right]$

Assuming that the motor current is balanced, the phase currents are

$i_{a}=-I_{s} \sin \theta, i_{b}=-I_{s} \sin \left(\theta-\frac{2 \pi}{3}\right), i_{c}=-I_{s} \sin \left(\theta+\frac{2 \pi}{3}\right)$

With (14) and (15), the fault circuit flux linkage equation is given by,

$$
\begin{aligned}
\lambda_{f}= & \left(L_{f}-\Delta\left(\frac{L_{m}}{2}+L_{l}\right)\right) i_{a}+\left(-L_{f}+2 \Delta\left(L_{m}+L_{l}\right)\right) i_{f} \\
& +\Delta \psi_{m} \cos \theta
\end{aligned}
$$

Considering the contract resistance $R_{f}$ and winding resistance $R$, the fault circuit dynamic equation is obtained such as 


$$
-\left(R_{f}+\Delta R\right) i_{f}+R_{f} i_{a}=\frac{d \lambda_{f}}{d t}
$$

Applying Laplace transform to the dynamic equation, the fault current $i_{f}$ can be given by

$$
\begin{aligned}
& \mathbf{I}_{f}(s)=\frac{c s+d \omega}{\left(s^{2}+\omega^{2}\right)(a s+b)} \\
& =\frac{(b c-a d \omega) s+(b d+c a \omega) \omega}{\left(s^{2}+\omega^{2}\right)\left(b^{2}+a^{2} \omega^{2}\right)}-\frac{a b c-a^{2} d \omega}{(a s+b)\left(b^{2}+a^{2} \omega^{2}\right)}
\end{aligned}
$$

where $a=-L_{f}+2 \Delta\left(L_{m}+L_{l}\right), \quad b=R_{f}+\Delta R$,

$$
c=\left(L_{f}-\Delta\left(\frac{L_{m}}{2}+L_{l}\right)\right) I_{s} \omega, \quad d=\Delta \psi_{m} \omega-R_{f} I_{s} .
$$

The fault current $i_{f}$ is given by

$$
\begin{aligned}
i_{f}= & \frac{b c-a d \omega}{a^{2} \omega^{2}+b^{2}} \cos \omega t+\frac{b d+a c \omega}{a^{2} \omega^{2}+b^{2}} \sin \omega t \\
& +\frac{a b c-a^{2} d \omega}{a^{2} \omega^{2}+b^{2}} e^{-b t / a} .
\end{aligned}
$$

Since second term on the right hand side of (19) is zero at steady, the fault current is obtained such as

$$
i_{f}=\frac{b c-a d \omega}{a^{2} \omega^{2}+b^{2}} \cos \omega t+\frac{b d+a c \omega}{a^{2} \omega^{2}+b^{2}} \sin \omega t
$$

The coupled flux linkage from $i_{f}$ to the healthy winding

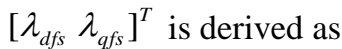

$$
\left.\begin{array}{rl}
{\left[\begin{array}{l}
\lambda_{d f s} \\
\lambda_{q f s}
\end{array}\right]=} & \frac{2}{3}\left(L_{f}-\Delta\left(\frac{L_{m}}{2}+L_{l}\right)\right) \\
& \times\left[\frac{b d+a c \omega}{b^{2}+a^{2} \omega^{2}} \cos \omega t+\frac{b c-a d \omega}{b^{2}+a^{2} \omega^{2}} \sin \omega t\right] \\
0
\end{array}\right] .
$$

From (21), the derivative of the flux linkages in the SRF $\left[\begin{array}{ll}\lambda_{d f e}^{+} & \lambda_{q f e}^{+}\end{array}\right]^{T},\left[\lambda_{d f e}^{-} \lambda_{q f e}^{-}\right]^{T}$ are derived as

$$
\begin{aligned}
& \frac{d}{d t}\left[\begin{array}{c}
\lambda_{d f e}^{+} \\
\lambda_{q f e}^{+}
\end{array}\right]=\frac{1}{3}\left(L_{f}-\Delta\left(\frac{L_{m}}{2}+L_{l}\right)\right) \frac{\omega}{b^{2}+a^{2} \omega^{2}}\left[\begin{array}{c}
b d+a c \omega \\
b c-a d \omega
\end{array}\right] \\
& \frac{d}{d t}\left[\begin{array}{l}
\lambda_{d f e}^{-} \\
\lambda_{q f e}^{-}
\end{array}\right]=\frac{1}{3}\left(L_{f}-\Delta\left(\frac{L_{m}}{2}+L_{l}\right)\right) \frac{\omega}{b^{2}+a^{2} \omega^{2}}\left[\begin{array}{c}
b d+a c \omega \\
-b c+a d \omega
\end{array}\right]
\end{aligned}
$$

(22) and (23) are coupling terms from the fault winding to the healthy abc-winding.

\section{Full model}

From (12), (13), (22), and (23), we obtain the full dynamic model of the PMSM under the a-phase winding turn short such that

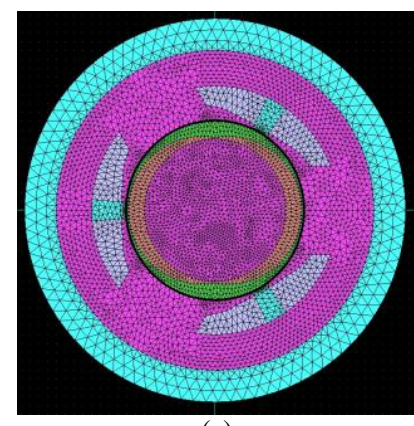

(a)

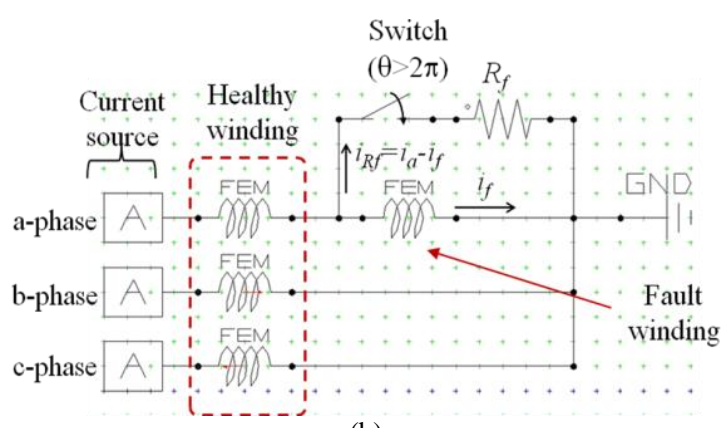

(b)

Fig. 3. (a) FEM simulation model and generated mesh of the PMSM with a-phase winding fault, (b) external circuit for the time based FEM simulation.

$$
\begin{aligned}
\mathbf{V}_{d q e}^{+} & =r \mathbf{I}_{d q e}^{+}+\frac{d}{d t} \lambda_{d q e}^{+} \\
= & r \mathbf{I}_{d q e}^{+}+\omega L_{S} \mathbf{J}_{d q e}^{+}+L_{S} \frac{d}{d t} \mathbf{I}_{d q e}^{+}+\omega\left(1-\frac{\Delta}{3}\right) \psi_{m}\left[\begin{array}{l}
0 \\
1
\end{array}\right] \\
& +\frac{\Delta_{m} \omega}{3} \mathbf{J I}_{d q e}^{+}+\frac{\Delta_{m} \omega}{3}\left[\begin{array}{ll}
0 & 1 \\
1 & 0
\end{array}\right] \mathbf{I}_{d q s}^{-}+\mathbf{L}_{\alpha} \frac{d \mathbf{I}_{d q e}^{+}}{d t}+p \boldsymbol{\lambda}_{f e}^{+}, \\
\mathbf{V}_{d q e}^{-} & =r \mathbf{I}_{d q e}^{-}+\frac{d}{d t} \lambda_{d q e}^{-} \\
= & r \mathbf{I}_{d q e}^{-}-\omega L_{S} \mathbf{J I}_{d q e}^{-}+L_{S} \frac{d}{d t} \mathbf{I}_{d q e}^{-}+\omega \frac{\Delta}{3} \psi_{m}\left[\begin{array}{l}
0 \\
1
\end{array}\right] \\
& -\frac{\Delta_{m} \omega}{3} \mathbf{J I}_{d q e}^{-}+\frac{\Delta_{m} \omega}{3}\left[\begin{array}{cc}
0 & -1 \\
-1 & 0
\end{array}\right] \mathbf{I}_{d q e}^{+}+\mathbf{L}_{\beta} \frac{d}{d t} \mathbf{I}_{d q e}^{-}+p \boldsymbol{\lambda}_{f e}^{-}
\end{aligned}
$$

If the negative sequence current controller regulates the negative sequence current $\mathbf{I}_{d q e}^{-}$to zero, the negative sequence voltage is calculated as

$$
\begin{aligned}
\mathbf{V}_{d q e}^{-} & =\omega \frac{\Delta}{3} \psi_{m}\left[\begin{array}{l}
0 \\
1
\end{array}\right]+\frac{\Delta_{m} \omega}{3}\left[\begin{array}{cc}
0 & -1 \\
-1 & 0
\end{array}\right] \mathbf{I}_{d q e}^{+} \\
& +\frac{1}{3}\left(L_{f}-\Delta\left(\frac{L_{m}}{2}+L_{l}\right)\right) \frac{\omega}{b^{2}+a^{2} \omega^{2}}\left[\begin{array}{c}
b d+a c \omega \\
-b c+a d \omega
\end{array}\right]
\end{aligned}
$$

Assuming that the three phase current is balanced and daxis current in the positive sequence SRF is zero, i.e., $I_{d e}^{+}=I_{d e}^{-}=I_{q e}^{-}=0$, the torque equation is obtained as follows 


$$
T=\psi_{m}\left[\left(1-\frac{\Delta}{3}+\frac{\Delta}{3} \cos 2 \theta\right) I_{q e}^{+}-\Delta i_{f} \sin \theta\right] .
$$

\section{Simulation Results}

To validate the model, FEM simulations are performed. System parameters are shown in Table 1. Fig. 3 shows a FEM mesh model and an external circuit model to make the a-phase turn short fault state. In Fig. 3(b), the a-phase is composed of two winding. The left one is healthy and the right one is faulted when the switch is turned on. Balanced three phase current sources are connected to the windings. At the beginning, the motor is healthy. The FEM simulation is conducted for the healthy state. After one rotor rotation $(\theta>2 \pi)$, the switch is turned on and the FEM simulation is conducted for the fault state.

Fig. 4 shows the comparison of the FEM simulation results and dynamic model simulation results when the motor speed is 3000rpm and the peak value of the balance phase current is 5A. To implement the dynamic model, (20), (24), (25), and (27) are used. In Fig. 4(a), $i_{f}$ of the FEM and the model results have different plots for $\theta<2 \pi$ since $i_{f}$ is assumed zero in the dynamic model but $i_{f}$ is the same with a-phase current in the FEM model. For $2 \pi<\theta<2 \pi+\pi / 2$, the dynamic model results are slightly different with the FEM. This comes from the transient element of the third term on the right hand side of (19). The time constant of the transient element is $5.5 \mathrm{msec}$ which is about $\pi / 2$ in electrical angle under 3000rpm. Hence, when $\theta>2 \pi+\pi / 2$, the dynamic model simulation results are almost identical to the FEM simulation results.

TABLE I

MOTOR SPECIFICATION

\begin{tabular}{|l|c|c|}
\hline \multicolumn{1}{|c|}{ Items } & Values & Units \\
\hline Pole number & 2 & \\
\hline Rated phase current & 5 & {$\left[\mathrm{~A}_{\text {peak }}\right]$} \\
\hline Rated phase to phase voltage & 220 & {$\left[\mathrm{~V}_{\text {rms }}\right]$} \\
\hline Self magnetizing inductance $\mathrm{L}_{\mathrm{m}}$ & 40 & {$[\mathrm{mH}]$} \\
\hline Leakage inductance $\mathrm{L}_{\mathrm{l}}$ & 10 & {$[\mathrm{mH}]$} \\
\hline $\mathrm{R}$ & 1.5 & {$[\Omega]$} \\
\hline $\mathrm{R}_{\mathrm{f}}$ & 0.5 & {$[\Omega]$} \\
\hline$\Delta$ & 0.33 & \\
\hline
\end{tabular}

Fig. 5 shows the negative sequence voltage vector in SRF with the FEM simulation results and (26). The calculation result from (26) has low difference with the FEM result. As the speed increase, the negative sequence voltage in the SRF increases. As the phase current increase, the negative sequence voltage vector rotates anticlockwise.

\section{Conclusion}

This paper has proposed the PMSM dynamic model under inter turn short which is derived in the positive and the negative sequence synchronous reference frames. With the proposed dynamic model, the fault detection scheme

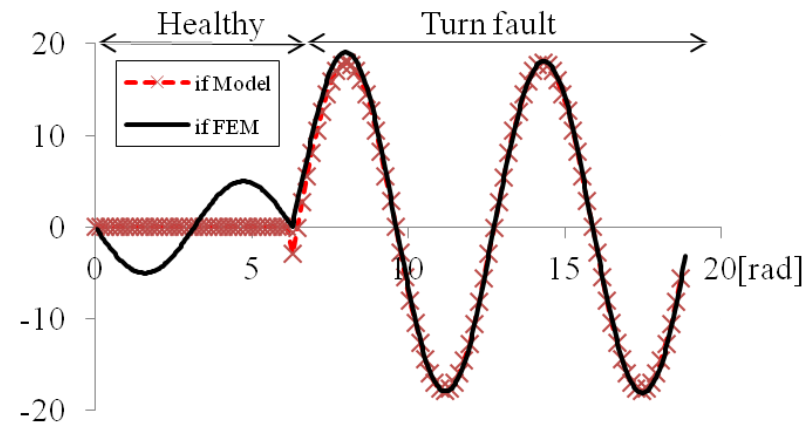

(a)

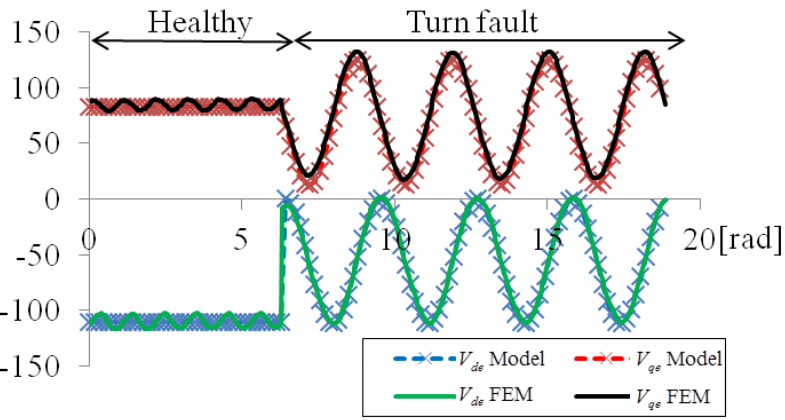

(b)

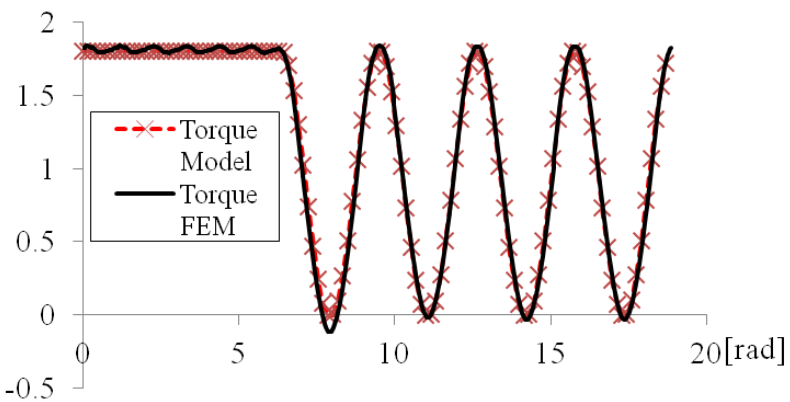

(c)

Fig. 4. Comparison of the FEM simulation and the dynamic model simulation when the motor speed is $3000 \mathrm{rpm}$ and the peak value of the balance phase current is $5 \mathrm{~A}$, (a) plot of $i_{\mathrm{f}}$ versus $\theta$, (b) plot of the motor voltages in the SRF versus $\theta$, (c) output torque versus $\theta$

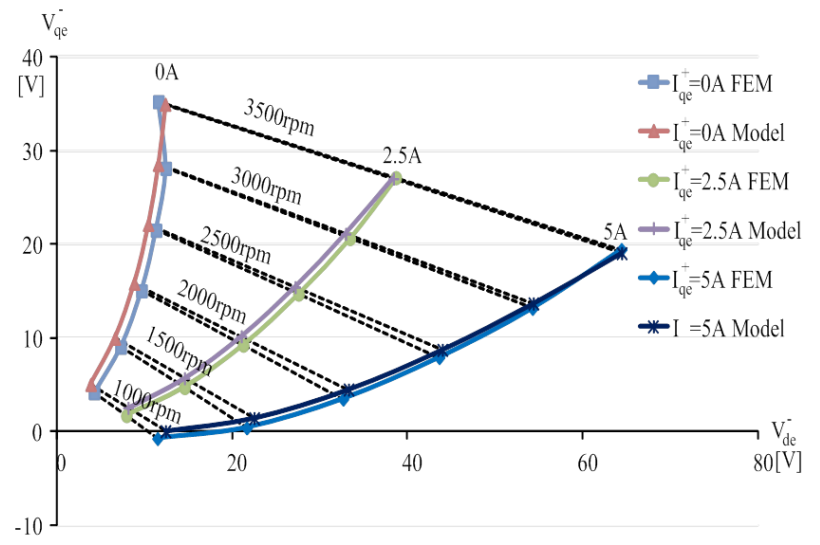

Fig. 5. Negative sequence voltage vector plots in SRF with the FEM simulation results and the proposed model. 
has been proposed also. To validate the proposed model, the FEM with electric circuit is conducted on the PMSM.

\section{Acknowledgement}

his work was supported by the "New \& Renewable Energy" of the Korea Institute of Energy Technology Evaluation and Planning(KETEP) grant funded by the Korea government Ministry of Knowledge Economy.

(No. 2012T100100064)

\section{References}

[1] I. Jeong, K. Nam, B. G. Gu. and I. S. Jung, "Dynamic modeling for PMSM with internal turn short fault," Power electronics and ECCE Asia (ICPE \& ECCE), pp. 2525-2528, 2011.

[2] A.L Nelson, M. Y. Chow, "Characterization of coil faults in an axial flux variable reluctance PM motor," IEEE Trans. Energy Conversion, vol. 17, no. 3, pp.340-348, 2002.

[3] Zhao, Y., Lipo, T.A., "Modeling and control of a multi-phase induction machine with structural unbalance," IEEE Trans. Energy Conversion, vol. 11, no. 3,pp.570-577, 1996.

[4] Adamiak, K., et al., "The cause and consequences of phase unbalance in single-sided linear induction motors," IEEE Trans. Magnetics, vol. 24, no. 6,pp.3223-3233, 1988.

[5] Taehyung Kim, et al., "The internal fault analysis of brushless DC motors based on the winding functioon theroy," IEEE Trans. Magnetics, vol. 45, no. 5,pp.2090-2096, 2009.

[6] J. Faiz, I. T. Ardekanei. And H. A. Toliyat, "An evaluation of inductances of a squirrel-cage induction motor under mixed eccentric conditions," IEEE Trans. Energy Conversion, Vol. 18, No. 2, June, 2003.

[7] T. Kim, H. W. Lee, and S. Kwak, "The internal fault analysis of brushless DC motors based on the winding function theory," IEEE Trans. Magnetics, Vol. 45, No. 5, May, 2009.

[8] B. Vaseghi, N. Takoraber, F. Meibdy-Tabar, A. Djedir, J-A. Farooq, A. Miraoui, "Modeling and characterizing the interturn short circuit fault in PMSM," IEEE IEMDC,pp.551-556, 2011. 